\title{
2007 Survey of Medical Toxicology Practice
}

\author{
Suzanne R. White • Beth Baker • Carl R. Baum • Anne Harvey • Robert Korte • \\ A. Nelson Avery $\cdot$ Lewis Nelson • Kevin Osterhoudt • Curtis Snook • Saralyn Williams
}

Published online: 31 March 2010

(C) American College of Medical Toxicology 2010

\begin{abstract}
To date, there appear to be no studies investigating the practice settings of all Medical Toxicology (MT) diplomates. The MT Assessment of Practice Performance Taskforce queried all MT diplomates about their current practice settings relative to the number of patients seen, the most common diagnoses, and the percent of time spent in their roles as medical toxicologists (MTs) and in their primary specialty. One hundred twenty-seven surveys were completed (44\% response rate). Seventy-nine percent of respondents were affiliated with poison centers. Eighty-eight percent of participants were clinically active and reported seeing or consulting on behalf of at least ten patients over a 2-year period. Acetaminophen toxicity was the most common diagnosis encountered by respondents. Other common diagnoses included antidepressant toxicity, antipsychotic toxicity, mental status alteration, metal/environmental toxicity, envenomation, and pesticide toxicity. While respondents were likely to spend more time in direct patient care in their primary specialty, compared to consulting on behalf of patients, they were more likely to consult on behalf of patients in their role as MTs. Respondents spent more time in research, education, and population health in their role as an MT than in their primary specialty. Administrative activities were more com-
\end{abstract}

\footnotetext{
S. R. White $(\triangle) \cdot$ A. Harvey $\cdot$ R. Korte $\cdot$ L. Nelson $\cdot$ C. Snook $\cdot$ S. Williams

American Board of Emergency Medicine, 3000 Coolidge Road,

East Lansing, MI 48823-6319, USA

e-mail: swhite1@dmc.org

B. Baker · A. N. Avery

American Board of Preventive Medicine,

Chicago, IL, USA

C. R. Baum $\cdot$ K. Osterhoudt

American Board of Pediatrics,

Chapel Hill, NC, USA
}

monly reported in association with the respondents' primary specialty than in their role as MTs. Most MTs encounter certain diagnoses with significant frequency and see a substantial number of patients within these categories. The majority spends more time on direct patient care in their primary specialty but is actively engaged in MT education, research, population health, and administration. A longitudinal assessment of MT practice patterns could inform MT curricular development and practice performance evaluation.

Keywords Board certification - Maintenance of certification . Practice performance assessment . Medical toxicology

\section{Introduction}

Medical Toxicology was recognized by the American Board of Medical Specialties (ABMS) in 1994. At the time of this inquiry, 287 diplomates (individuals who have achieved ABMS board certification) were certified in this relatively new subspecialty that is sponsored by the boards of emergency medicine, pediatrics, and preventive medicine. To date, there appear to be no studies investigating the practice settings of all Medical Toxicology diplomates. One study looked at a segment of the population, 94 fellowship trainees, and found that while $91 \%$ of the respondents remained active in medical toxicology, $78 \%$ spent less than three fourths of their professional time in toxicology-related activities [1]. No study has assessed the whole population relative to patient interaction or other practice issues. This information could have important relevance to the specialty and beyond. Information about medical toxicologists who see or consult on behalf of either individual patients or populations would be helpful to medical toxicology training programs and to certifying or licensing bodies as they develop training curricula and practice evaluation tools. 
Medical Toxicology Assessment of Practice Performance Survey Questions

\section{Practice Setting}

Questions 1 through 3 are designed to obtain a picture of the various types of practice settings in which medical toxicologists work. You are asked to estimate aspects of your work in terms of numbers of patients and percentages of time performing various tasks. Medical Toxicology patients are defined as those individual patients with whom you have had direct or indirect care relative to The Core Content of Medical Toxicology. These numbers may vary across time and setting; however, please provide your best estimates.

1. How many individual Medical Toxicology patients have you treated directly or consulted with others about their direct care over the past two years?

\begin{tabular}{lcc} 
& \multicolumn{2}{c}{ Individual Patients } \\
None & Treated & Consulted \\
$1-10$ & 0 & 0 \\
$11-20$ & 0 & 0 \\
$21-50$ & 0 & 0 \\
$51-100$ & 0 & 0 \\
$101-200$ & 0 & 0 \\
More than 200 & 0 & 0 \\
& 0 & 0
\end{tabular}

2. What are the three most common diagnoses of Medical Toxicology patients you have treated or consulted about in the last two years? Please indicate the approximate number of Medical Toxicology patients with whom you have had direct or indirect contact for each diagnosis.

Diagnosis

\begin{tabular}{cccccc}
\multicolumn{7}{c}{ Number of Patients } \\
$1-10$ & $11-20$ & $21-50$ & $51-100$ & $101-200$ & More than 200 \\
0 & 0 & 0 & 0 & 0 & 0 \\
0 & 0 & 0 & 0 & 0 & 0 \\
0 & 0 & 0 & 0 & 0 & 0
\end{tabular}

3. Consider the amount of time you spend in your professional activities. How much time do you spend in Medical Toxicology and your Primary specialty? Estimate the percentage of time you spend in the following activities for both your work in Medical Toxicology and your primary specialty.

$\underline{\text { Activity }}$

$\%$ of Time
Medical Toxicology
Primary Specialty

Direct patient care

Individual patient consultation

Population health

Administration

Research

Education

Other (Please Specify)

Fig. 1 Medical toxicology assessment of practice performance survey questions 
Table 1 Respondents by primary board

\begin{tabular}{lrrrrr}
\hline Primary board & \multicolumn{2}{c}{ Population } & & \multicolumn{2}{l}{ Sample } \\
\cline { 2 - 3 } \cline { 5 - 6 } & No. & $\%$ & & No. & $\%$ \\
\hline ABEM & 236 & 82 & & 97 & 80 \\
ABPM & 29 & 10 & & 15 & 12 \\
ABP & 22 & 8 & & 9 & 7 \\
Unknown & & & $6^{\text {a }}$ & \\
Total & 287 & & 127 & \\
\hline
\end{tabular}

${ }^{a}$ Due to program error on the first day of the survey, the primary boards of six respondents were not captured. The six are not listed as part of the percent returned by primary specialty

\section{Methods}

In 2007, the Medical Toxicology Maintenance of Certification Task Force, on behalf of the Medical Toxicology Subboard, developed and conducted an online survey of all Medical Toxicology diplomates asking about their current practice setting. The survey asked about physicians' practice settings in terms of the number of individual patients they saw, patients on whose behalf they consulted, the most common diagnoses, and the percent of time spent in their roles as a medical toxicologist and in their primary specialty. The final survey, which was administered via the Internet, is presented in Fig. 1. A letter was sent to all 287 diplomates with the Internet address of the survey, their ID, and password. On October 26, 2007, a reminder postcard was sent to diplomates who had not yet completed the survey. The study was granted IRB Exemption (protocol 0905007159, Wayne State University Human Investigation Committee).

\section{Results}

One hundred twenty-seven surveys (44\%) were completed. This is similar to response rates observed with most social science surveys [2]. Others have noted lower physician responses to surveys than for other groups. For example, a comparable study by Jepson et al. obtained a response rate of $38 \%$ when mailing questionnaires to a sample of generalist physicians [3].

\section{Respondents by Primary Board}

The percent of diplomates in the population and the sample of diplomates who submitted surveys are presented by primary board in Table 1. Eighty percent (80\%) of respondents listed their primary board as emergency medicine, $12 \%$ preventive medicine, and $7 \%$ pediatrics. These results indicate that the primary specialty of the sample of survey respondents is similar to that of the total population of Medical Toxicology diplomates: emergency medicine $(82 \%)$, preventive medicine $(10 \%)$, and pediatrics $(8 \%)$. Due to a program error on the first day of the survey, the primary boards of six respondents were not captured. The six are therefore not listed as part of the response rate by primary specialty.

\section{Practice Setting}

The first section of the survey asked about participants' practice settings and the types of activities in which they are engaged. Seventy-nine percent of respondents are affiliated with poison centers. Participants were further asked to estimate the number of patients they see with specific diagnoses and the percent of their professional time spent in a variety of activities in the role of a medical toxicologist and as a practitioner in their primary specialty. Results indicated that $95 \%$ of respondents either treat or consult on behalf of individual patients, with $88 \%$ seeing or consulting on behalf of greater than ten patients over a 2-year period.

\section{Most Common Diagnoses}

One hundred twenty-five respondents listed their three most common diagnoses. These results are listed in Table 2. Acetaminophen toxicity was most common, with $62 \%$ of respondents listing it among their top three. Other common diagnoses included antidepressant toxicity, antipsychotic toxicity, mental status alteration, metal/environmental toxicity, snake or other envenomation, and pesticide toxicity. Respondents were further asked to indicate the number of patients they saw or on whose behalf they consulted for each of these diagnoses over the past 2 years. The purpose of this question was to determine whether diplomates saw enough patients in a single diagnosis category that would allow pooling of patient data to evaluate trends in practice performance. The results displayed in Table 3 indicate that

Table 2 Most common diagnoses in medical toxicology practice

Number of diagnoses included in top three $(N=127)$

\begin{tabular}{lll}
\hline Diagnosis & $\begin{array}{l}\text { Number of } \\
\text { respondents } \\
\text { citing diagnosis }\end{array}$ & $\begin{array}{l}\text { Percent of } \\
\text { respondents } \\
\text { citing diagnosis }\end{array}$ \\
\hline
\end{tabular}

\begin{tabular}{lrr}
\hline Acetaminophen toxicity & 67 & $53 \%$ \\
Antidepressant toxicity & 17 & $13 \%$ \\
Antipsychotic toxicity & 15 & $12 \%$ \\
Mental status alteration & 14 & $11 \%$ \\
Metal/environmental & 10 & $8 \%$ \\
$\quad$ toxicity & 10 & $8 \%$ \\
Snake envenomation & 5 & $4 \%$ \\
\hline
\end{tabular}


Table 3 Number of patients by selected diagnosis

\begin{tabular}{|c|c|c|c|c|c|c|}
\hline \multirow[t]{2}{*}{ Diagnoses } & \multicolumn{6}{|c|}{ Number of Patients } \\
\hline & $1-10$ & $11-20$ & $21-50$ & $51-100$ & $101-200$ & $>200$ \\
\hline 1 & $10 \%$ & $14 \%$ & $22 \%$ & $22 \%$ & $10 \%$ & $22 \%$ \\
\hline 2 & $11 \%$ & $20 \%$ & $28 \%$ & $23 \%$ & $7 \%$ & $11 \%$ \\
\hline 3 & $15 \%$ & $22 \%$ & $29 \%$ & $17 \%$ & $8 \%$ & $8 \%$ \\
\hline
\end{tabular}

the majority of diplomates see or consult on behalf of a substantial number of patients within each of their top three identified diagnoses.

Percent of Time Spent in Medical Toxicology and Primary Specialty

The results were analyzed by determining the percent of respondents who spend more time in either Medical Toxicology or primary specialty activities. Sixty-five percent of respondents spent more time on direct patient care in their primary specialty than they spent on direct patient care in Medical Toxicology. The results are shown in Table 4. While respondents were likely to spend more time in direct patient care in their primary specialty, they were more likely to consult on behalf of patients in their role as a medical toxicologist than in their primary specialty role. In general, respondents spent more time in research, education, and population health in their role as a medical toxicologist than in their primary specialty role. Administrative activities were more commonly reported in association with the respondents' primary specialty than in their role as medical toxicologists.

\section{Discussion}

The scope of practice for the entire population of medical toxicology diplomates relative to patient interaction or other practice issues has not previously been assessed. Our survey was designed to examine this uncertainty. The use of an online survey facilitated sampling, and the response rate achieved was acceptable. As important as the survey response rate, however, is the assurance that the sample completing the survey is similar to the total population to which inferences are being made. For the purpose of this study, one of the greatest potential variations in the survey population is work setting, which is represented here by the diplomates' primary board. Since this sample of respondents mirrored the work setting for the entire population of medical toxicology diplomates, it is representative of the whole population.

Most medical toxicologists are clinically active as defined by the American Board of Medical Specialties' guidelines, i.e., as any amount of direct or consultative patient care provided within the past 24 months. In fact, $95 \%$ of respondents either treat or consult on behalf of individual Medical Toxicology patients, with $88 \%$ seeing or consulting on behalf of 11 or more patients over a 2 -year period. Most respondents were able to list their three most common Medical Toxicology diagnoses encountered. Acetaminophen toxicity was most common, with $62 \%$ of respondents listing it among their top three. This finding is consistent with another report from the same year that implicated acetaminophen in $27 \%$ of poisoning fatalities reported to the 2007 American Association of Poison Control Centers' National Poisoning Data System (NPDS) [4]. It is expected that medical toxicologists would likely be consulted to assist with managing these types of critically ill, poisoned patients. Other common diagnoses reported by medical toxicologist respondents parallel those substances reported with significant frequency to NPDS. The majority of diplomates see or consult on behalf of a substantial number of patients within each of their identified top three diagnoses. This suggests some degree of consistency
Table 4 Percent of time spent in medical toxicology and primary specialty

\begin{tabular}{lccc}
\hline Activity & \% Spending more time & \\
\cline { 2 - 4 } & Medical toxicology & Equal time & Primary specialty \\
\hline Research & 65 & 16 & 18 \\
Patient consultation & 60 & 10 & 30 \\
Education & 56 & 23 & 21 \\
Population health & 50 & 19 & 31 \\
Administration & 39 & 10 & 50 \\
Direct patient care & 23 & 12 & 65 \\
Other & 38 & 42 & 19 \\
\hline
\end{tabular}


relative to the types of diagnoses encountered in medical toxicology practice.

Sixty-five percent of respondents spent more time on direct patient care in their primary specialty rather than Medical Toxicology but were more likely to consult on behalf of patients in their role as a medical toxicologist. This makes logical sense given the traditional role of medical toxicologists and that $79 \%$ of respondents are affiliated with poison control centers. One limitation of these results, however, is that the questions relating to the percent of time across professional activities spent as a medical toxicologist and as a practitioner in their primary specialty were interpreted two ways by respondents; some respondents divided their total time within categories and part across categories. To a degree, this may have confounded the results.

There are limitations to this study. In general, surveys have a number of limitations relative to the validity and reliability of responses obtained to questions, including recall bias. We believe that the validity of the results of this survey was optimized through its development by medical toxicologists from each of the three sponsoring primary specialties and by their familiarity with the group being studied. This allowed the application of knowledge about the norms and values of this group in preparing the survey. The questions relating to the percent of time across activities spent as a medical toxicologist and as a practitioner in their primary specialty were interpreted two ways by respondents; some respondents divided their total time within categories and part across categories. To a degree, this confounded the results. There was no attempt to verify the results reported online by respondents. Another limitation is the potential for selection bias. Since demographic information on the nonrespondents is not available, there are many potential factors that could have impacted their likelihood to respond, such as length of time in practice and attitude toward credentialing processes in general, to name a few. Finally, this survey was meant to primarily assess the current clinical practice of medical toxicology and not to define the entire scope of medical toxicology activities, many of which are nonclinical. None- theless, these data may be informative to those developing medical toxicology practice assessment programs.

In conclusion, the practice characteristics for medical toxicology diplomates was assessed. Most medical toxicologists are clinically active. They encounter certain diagnoses with significant frequency and see a substantial number of patients within these categories. These diagnoses include acetaminophen toxicity, psychiatric medication toxicity, pesticide toxicity, and environmental exposures. Furthermore, medical toxicologists are frequently consulted for patients with altered mental status. The majority spends more time on direct patient care in their primary specialty, rather than Medical Toxicology, but is more likely to consult on behalf of patients in their role as a medical toxicologist. Medical toxicologists are actively engaged in other activities such as education, research, population health, and administration. They are more likely to spend time in medical toxicology education, research, and population health compared to their primary specialty. A longitudinal assessment of medical toxicology practice patterns could inform medical toxicology training programs as they develop educational curricula. Furthermore, a longitudinal assessment could be useful to certifying or licensing bodies as they develop practice performance evaluation tools.

\section{References}

1. Wax PM, Donovan JW (2000) Fellowship training in medical toxicology: characteristics, perceptions and career impact. J Toxicol Clin Toxicol 38(6):637-642

2. Johnson T, Owens L (2003) Survey response rate reporting in the professional literature. In: Proceedings from the 58th Annual Meeting of the American Association for Public Opinion Research, Section on Survey Research Methods. Nashville, TN, May 2003, pp 127-133

3. Lepson C, Asch DA, Hershey JC, Ubel PA (2005) I a mailed physician survey, questionnaire length had a threshold effect on response rate. J Clin Epidemiol 58(1):103-105

4. Bronstein AC, Spyker DA, Cantilena LR, Green JL, Rumack BH, Heard SE (2008) 2007 Annual report of the American association of poison control centers' national poison data system (NPDS): 25th annual report. Clin Toxicol 46:927-1057 